\title{
A pandemia de COVID-19 e a saúde mental de idosos: possibilidades de atividade física por meio dos Exergames
}

\author{
The COVID-19 pandemic and the mental health of the elderly: possibilities of \\ physical activity through Exergames
}

\section{AUTORES \\ Saulo Vasconcelos Rocha ${ }^{1}$ (iD Carolina Rego Chaves $\operatorname{Dias}^{2}$ (D) Mônica Costa Silva ${ }^{3}$ (D) \\ Camilo Luis Monteiro Lourenço ${ }^{3}$ (D) \\ Clarice Alves dos Santos ${ }^{1}$ (D) \\ 1 Universidade Estadual do Sudoeste da Bahia Programa de Pós-Graduação em Educação Física UESB/UESC, Jequié, Bahia, Brasil. \\ 2 Universidade Estadual do Sudoeste da Bahia, Departamento de Saúde II, Jequié, Bahia, Brasil. 3 Universidade Federal de Santa Catarina, Departamento de Educação Física, Florianópolis, Santa Catarina, Brasil. \\ CONTATO}

Saulo Vasconcelos Rocha

svrocha@uesb.edu.br

Avenida José Moreira Sobrinho, S/N, Je-

quiezinho, Jequié, Bahia, Brasil.

CEP: 45200-000.

DOI

$10.12820 /$ rbafs. $25 \mathrm{e} 0142$

\section{(cc) BY}

Este trabalho está licenciado com uma Licença

Creative Commons - Atribuição 4.0 Internacional.

\begin{abstract}
RESUMO
Este ensaio teórico foi elaborado com o objetivo de discutir sobre os reflexos da pandemia na saúde mental de idosos e a contribuição dos exergames como terapia não medicamentosa para o cuidado à saúde neste grupo em tempos de pandemia de COVID-19. Apesar de compreender que as medidas restritivas para aglomerações são estratégias importantes no controle da pandemia, o distanciamento social pode aumentar ainda mais a vulnerabilidade dos idosos, principalmente no que diz respeito ao agravamento das morbidades psíquicas. Nesse sentido, apresentar possibilidades de atividades físicas, já comprovadamente aceitas, como modalidades terapêuticas efetivas, frente a essas condições, e indicar possibilidades de movimentar-se com os exergames, contribui para o aumento do nível de atividade física e, consequentemente, para a melhoria da saúde mental dessa população.
\end{abstract}

Palavras-chave: Atividade motora; Idoso; Saúde mental; Infecções por coronavírus.

ABSTRACT

This theoretical essay was prepared with the aims to discuss the effects of the pandemic on mental health of elderly people and the contribution of exergames as non-drug therapy for health care in this group in times of the COVID-19 pandemic. Despite understanding that restrictive measures for agglomerations are important strategies in controlling the pandemic, social isolation can further increase the vulnerability of the elderly, especially the worsening of psychic morbidities. In this sense, presenting possibilities of physical activities, already proven accepted, as effective therapeutic modalities, in the face of these conditions, and indicating possibilities of moving with exergames, contributes to the increase in the level of physical activity and, consequently, to the improvement mental health of this population.

Keywords: Motor activity; Elderly; Mental health; Coronavirus infections.

\section{Introdução}

A pandemia causada pelo SARS-CoV-2, popularmente conhecido como COVID-19, se espalhou pelo mundo de forma rápida, devido à sua alta capacidade de transmissibilidade. $\mathrm{Na}$ tentativa de conter esse novo coronavírus, a Organização Mundial de Saúde (OMS) orientou algumas estratégias de prevenção, de acordo com a avaliação de risco de cada localidade, como evitar aglomerações, cancelamentos de eventos sociais, fechamento de comércios, restrições de viagens e o distanciamento social ${ }^{1}$.
O distanciamento social é a estratégia mais eficiente para evitar o contágio, mas pode trazer prejuízos psicológicos à população $0^{2,3,4}$. Além disso, pode provocar um aumento no comportamento sedentário e na inatividade física, sendo que ambas condições estão atreladas às doenças física, mental e maior o risco de morte, principalmente em populações mais vulneráveis, em especial os idosos ${ }^{5,6}$.

Embora haja uma preocupação para que os idosos permaneçam em casa, por apresentarem maior vulnerabilidade quando infectados pela COVID-19, níveis insuficientes de atividade física podem apresentar agra- 
vos na saúde mental e emocional nessa população ${ }^{7}$. Por outro lado, a atividade física é apontada como apoio fundamental na saúde física e mental, além de promover efeitos adicionais positivos no processo de envelhecimento e em doenças associadas ${ }^{8}$.

Diante do efeito deletério que o isolamento social possa ocasionar na população idosa, faz-se necessário pensar em estratégias para realizações de atividades físicas em casa, a fim de minimizar as consequências nas saúdes física e mental de idosos durante a pandemia. Neste sentido, o objetivo do presente ensaio é discutir sobre os reflexos da pandemia na saúde mental de idosos e a contribuição dos exergames como terapia não medicamentosa para o cuidado à saúde neste grupo em tempos de pandemia de COVID-19.

\section{Isolamento social, vulnerabilidade e saúde mental dos idosos}

A COVID-19 é uma doença respiratória infectocontagiosa, que pode apresentar infecções assintomáticas e infecções respiratórias graves ${ }^{2}$. A maioria das pessoas pode ser assintomática, e não necessita de atendimento hospitalar, porém algumas podem desenvolver infecções agudas graves com maior probabilidade de morte, principalmente os idosos.

As pessoas idosas, especialmente as mais longevas, fazem parte do grupo de maior risco para a COVID-19, devido à maior exposição a comorbidades que estão associadas ao aumento da letalidade pela doença ${ }^{6,9}$. Nesse contexto, é imprescindível que essa população mantenha-se em distanciamento social, evitando o máximo de contato físico, até mesmo com familiares. Embora essa medida seja importante para proteção da saúde, sobretudo para os idosos, este pode implicar consequências adversas em outras situações de saúde, que podem perdurar mesmo depois do fim da pandemia ${ }^{7}$.

O distanciamento, por si, torna-se uma atitude favorável para alterações comportamentais dos idosos, visto que estes encontram-se mais vulneráveis aos determinantes sociais da saúde que podem favorecer o aparecimento de doenças ou agravar as pré-existentes. Nesse sentido, as atividades que envolvem interações sociais são intervenções importantes para a saúde e o bem-estar dos idosos ${ }^{10}$. A redução dessas práticas implica grandes desafios, sobretudo para a manutenção do envelhecimento ativo.

Dentre os efeitos psicológicos que o distanciamento social pode desencadear neste grupo etário estão a insônia, o medo de ser contaminado, a ansiedade, as preocupações com os seus entes queridos e a frustração por não saber quando a situação será controlada. Ressalta-se que as informações insuficientes e a dificuldade financeira podem contribuir para o aumento desses efeitos psicológicos ${ }^{7}$.

Aspectos como aumento da solidão e a redução das interações sociais são fatores de risco para vários transtornos mentais, como a depressão geriátrica ${ }^{3}$. Outras consequências aos estados psicológicos são os ataques de pânico, o transtorno de estresse pós-traumático, os sintomas psicóticos e o suicídio ${ }^{4}$.

Em contrapartida, o bem-estar psicológico pode ser potencializado, a partir do momento que os idosos são estimulados, mesmo que em suas residências, a realizarem algumas atividades físicas ${ }^{9}$, atividades de memória, assim como aproximar-se com a realidade virtual, como processo de educação continuada, através do uso do storytteling ${ }^{11}$.

Por isso, é importante identificar as condições biopsicossociais de cada idoso, além de realçar as suas vulnerabilidades, no sentido de promover estratégias que possam contribuir para uma senescência ativa. Dentre as estratégias, a utilização dos exergames, como modalidade de atividade física, apresenta-se como uma alternativa terapêutica importante no contexto de pandemia pela COVID-19.

\section{Atividade física em casa por meio de exergames: uma possibilidade divertida $\mathrm{e}$ eficaz}

Como citado anteriormente, o distanciamento social é uma medida necessária no processo de combate à rápida disseminação do SARS-CoV-2. A exposição a essa condição está diretamente associada ao decréscimo nos níveis de atividade física em qualquer intensidade (vigorosa, moderada e leve) concomitante à elevação do tempo sedentário ${ }^{12}$. Situações que podem agravar ainda mais problemas de natureza psicoemocionais, como níveis mais elevados de estresse e de ansiedade, os quais podem ser potencializados com a pandemia da $\mathrm{CO}$ VID-19 e com o confinamento dela decorrente ${ }^{13}$. Os efeitos psicológicos negativos e a redução nos níveis de atividade física podem gerar consequências até mesmo depois do fim da pandemia ${ }^{5,6}$.

Nos grupos de indivíduos com múltiplas comorbidades e nos psicologicamente mais vulneráveis, a exemplo dos idosos, isso torna-se ainda mais preocupante, pois é justamente nesses grupos que se espera haver maior severidade e taxa de letalidade por COVID-19. 
Diante dessas informações, é plausível supor que exista um ciclo vicioso envolvendo o autoisolamento e o agravamento de problemas psicoemocionais. Desse modo, faz-se necessário pensar e adotar possibilidades de confrontar os crescentes problemas psicoemocionais oriundos $\mathrm{da} / \mathrm{na}$ quarentena durante a pandemia de COVID-19, além de buscar estratégias para reduzir os riscos associados à forma mais grave da doença.

Alinhado a esse propósito, a atividade física tem reconhecido papel positivo sobre as diferentes dimensões da saúde das pessoas, cujos benefícios encontram-se descritos na literatura. Ter a saúde mental equilibrada é um desses benefícios provenientes da prática regular de atividade física. Entretanto, em tempos de pandemia por COVID-19, torna-se inviável e mais arriscado para os grupos populacionais, especialmente para os idosos, acessarem os ambientes cotidianos da prática de atividades físicas, como as academias, os parques públicos ou as atividades oferecidas nos grupos de exercícios em centros de saúde. Nesse contexto, as atividades físicas realizadas no ambiente domiciliar são uma possibilidade estratégica dos idosos alcançarem dois objetivos com uma mesma ação: cumprir com o requisito da quarentena, enquanto mantêm o nível recomendado de atividade física para a saúde.

É nessa perspectiva que se encaixam os exergames. Eles são um tipo de jogo de videogame não sedentário, o qual requer esforço físico do participante para ser jogado. Os exergames têm se apresentado como uma possibilidade divertida e eficaz de promover atividade física em casa. Usualmente, os exergames são jogados por meio de plataformas comerciais de jogos eletrônicos, o que pode facilitar o acesso das pessoas para terem esse recurso tecnológico em suas residências.

Uma gama de atividades físicas pode ser realizada por meio dos exergames, as quais variam de acordo com o pacote e a plataforma de jogo. Desde atividades esportivas tradicionais, mimetizando o futebol, tênis de quadra e de mesa, vôlei de praia, natação e atletismo (lançamento de disco, arremesso de dardo, corrida com barreiras etc.), exercícios de academia (exercícios de calistenia e alongamento, corrida, treinamento de força etc.), yoga, boxe, esportes de aventura (descida de corredeiras, jogos espaciais etc.), exercícios de equilíbrio, dança (Zumba, dança de rua) entre outros. Uma importante característica desses videogames ativos é a possibilidade de desempenhá-los de modo individual ou múltiplo-jogador, pois o modo de jogo tem efeito sobre o gasto energético - jogar com/contra alguém pode ser mais intenso, facilitar a troca de experiências e possibilitar a amizade e mediar benefícios psicológicos ${ }^{14}$.

Ao pensar que o arranjo familiar brasileiro proporciona que a quarentena do idoso esteja sendo feita com a presença de seus filhos, companheiros ou outro familiar, os exergames podem ser compartilhados para uma experiência divertida em família. A literatura tem demonstrado que intervenções com exergames impactam positivamente na qualidade de vida e sintomas da depressão em adultos mais velhos e, nesse momento que os idosos se encontram reclusos, em isolamento/ distanciamento social, para cumprir a quarentena, os exergames podem ser uma ferramenta interessante para promoção da atividade física na busca por uma saúde mental equilibrada ${ }^{14,15}$.

Cabe salientar que apesar da extensa literatura acerca dos efeitos dos exergames na saúde das pessoas, em especial nos desfechos relativos à saúde mental ${ }^{14,15}$, as intervenções realizadas com esse tipo de videogames ativos foram realizadas em um contexto "pré-pandemia”. Nesse sentido, são necessários estudos de intervenção com exergames para avaliar seus efeitos sobre aspectos da saúde mental de idosos durante o período de pandemia.

No cenário de pandemia, no qual medidas de distanciamento são essenciais para a proteção da saúde da população idosa, manter os níveis recomendados da prática de atividade física e reduzir o comportamento sedentário são medidas importantes para a melhora da saúde mental.

Dentre as modalidades de atividade física, os exergames apresentam-se como uma estratégia viável, lúdica e que permite a socialização, mesmo com o distanciamento físico, e que pode ser utilizada dentro do contexto atual de pandemia vivenciado por todos, proporcionando um aumento do nível de atividade física e uma melhor sensação de bem-estar aos participantes.

\section{Conflito de interesse}

Os autores declaram não haver conflito de interesse.

\section{Contribuição dos autores}

Rocha SV, participou da concepção e redação do manuscrito e revisão crítica dos conteúdos. Dias CRC, participou da concepção e redação do manuscrito. Silva $\mathrm{MC}$, participou da concepção e redação do manuscrito. Lourenço CLM, participou da concepção e redação do manuscrito. Santos CA, participou da concepção e redação do manuscrito. 


\section{Referências}

1. World Health Organization. Considerations in adjusting public health and social measures in the context of COVID-19: interim guidance. Geneva, Switzerland: World Health Organization; 2020. [citado em 2020 ago 21]. Disponível em: https://www.who.int/publications/i/ item/considerations-in-adjusting-public-health-and-socialmeasures-in-the-context-of-covid-19-interim-guidance

2. Usher K, Bhullar N, Jackson D. Life in the pandemic: Social isolation and mental health. J Clin Nurs. 2020;29(1516):2756-7.

3. Fiorillho A, Gorwood P. The consequences of the COVID-19 pandemic on mental health and implications for clinical practice. Eur Psychiatry. 2020;63(1):e32.

4. Pereira MD, Oliveira LC, Costa CFT, Bezerra CMO, Pereira MD, Santos CKA, et al. The COVID-19 pandemic, social isolation, consequences on mental health and coping strategies: an integrative review. Res Soc Dev. 2020;9(7):1-35.

5. Booth FW, Roberts CK, Thyfault JP, Ruegsegger GN, Toedebusch RG. Role of inactivity in chronic diseases: evolutionary insight and pathophysiological mechanisms. Physiol. Rev. 2017;97(4):1351-402.

6. Jiménez-Pavón D, Carbonell-Baeza A, Lavie CJ. Physical exercise as therapy to fight against the mental and physical consequences of COVID-19 quarantine: Special focus in older people. Prog Cardiovasc Dis. 2020;63(3):386-8.

7. Brooks SK, Webster RK, Smith LE, Woodland L, Wessely S, Greenberg N, et al. The psychological impact of quarantine and how to reduce it: rapid review of the evidence. The Lancet. 2020;395(issue 10227):912-20.

8. Cadore EL, Asteasu MLS, Izquierdo M. Multicomponent exercise and the hallmarks of frailty: Considerations on cognitive impairment and acute hospitalization. Exp Gerontol. 2019;122:10-14.
9. Rodríguez MA, Crespo I, Olmedillas H. Exercising in times of COVID-19: what do experts recommend doing within four walls? Rev Esp Cardiol (Engl Ed). 2020;73(7):527-9.

10. Ferreira HG, Barham EJ. O Envolvimento de idosos em atividades prazerosas: Revisão da Literatura sobre Instrumentos de Aferição. Rev Bras Geriatr Gerontol. 2011;14(3):579-90.

11. Costa NP, Polaro SHI, Vahl EAC, Gonçalves LHT. Storytelling: a care technology in continuing education for active ageing. Rev Bras Enferm. 2016;69(6):1068-75.

12. Ammar A, Brach M, Trabelsi K, Chtourou H, Boukhris O, Masmoudi L, et al. Effects of COVID-19 Home Confinement on Eating Behaviour and Physical Activity: Results of the ECLB-COVID19 International Online Survey. Nutrients. 2020;12(6):1583.

13. Ozamiz-Etxebarria N, Dosil-Santamaria M, PicazaGorrochategui M, Idoiaga-Mondragon N. Stress, anxiety, and depression levels in the initial stage of the COVID-19 outbreak in a population sample in the northern Spain. Cad Saúde Pública. 2020;36(4):e00054020.

14. Lee S, Kim W, Park T, Peing W. The Psychological Effects of Playing Exergames: A Systematic Review. Cyberpsychol Behav Soc Netw. 2017;20(9):513-32.

15. Andrade A, Correia CK, Coimbra DR. The Psychological Effects of Exergames for Children and Adolescents with Obesity: A Systematic Review and Meta-Analysis. Cyberpsychol Behav Soc Netw. 2019;22(11):724-35.

Recebido: 29/08/2020

Aprovado: 09/10/2020 